\title{
A new portable ELF Schumann resonance receiver: design and detailed analysis of the antenna and the analog front-end
}

\author{
Constantinos I. Votis ${ }^{1}$, Giorgos Tatsis ${ }^{1}$, Vasilis Christofilakis', Spyridon K. Chronopoulos ${ }^{1 *}$ (D), Panos Kostarakis ${ }^{1}$, \\ Vasilis Tritakis $^{2}$ and Christos Repapis ${ }^{2}$
}

\begin{abstract}
Schumann resonance oscillation detection is a complex procedure which requires customized and high-quality measurement systems. The primary objective of this work was to design and implement a stand-alone, portable, and low-cost receiver able to measure as much Schumann resonance harmonics as possible. Design, as well as detailed analysis of the efficient induction coil magnetic antenna and the low-noise amplifying-filtering chain, is presented. The detection system includes two coils back to back, resulting in a total coil length of $60 \mathrm{~cm}$. The filtering and amplification chain exhibits an experimentally measured total passband gain equal to $112 \mathrm{~dB}$ at $10 \mathrm{~Hz}$ and as low as $2.88 \mathrm{nV} / \sqrt{ } \mathrm{Hz}$ equivalent input noise. In order to validate the new portable ELF Schumann resonance detection and monitoring system, we took measurements at various spots "relatively" free from man-made electromagnetic pollution. Results have shown very clear Schumann resonance peaks for the first six modes with 10-min acquisition time.
\end{abstract}

Keywords: Receiving antenna, Analog front-end, Amplifier, Filter, System testing, Schumann resonance measurements

\section{Introduction}

The Schumann resonances (SR) are electromagnetic oscillations of the Earth-ionosphere cavity at frequencies of $7.8,14,20,26,33,39$, and $45 \mathrm{~Hz}$. The long-term monitoring of the Schumann phenomenon has recently drawn attention, not least from the space-geophysics community [1-3]. SR measurements and analysis provide information on the planetary thunderstorm activities, the properties of lower ionosphere layers, the Earth surface and atmosphere temperature variations, and the properties of earthquakes as well as on the studies of other celestial bodies [4-9]. Consequently, increased interest has been shown by the scientific community on the methods and techniques employed in SR experimental detection. The detection of SR is a complex procedure that employs the limited energy generated and dissipated by the global lighting activity. This total

\footnotetext{
* Correspondence: schrono@cc.uoi.gr; spychro@gmail.com

${ }^{1}$ Electronics-Telecommunications and Applications Lab Physics Department, University of loannina, 45110 loannina, Greece

Full list of author information is available at the end of the article
}

energy is then smeared inside the huge volume of the Earth-ionosphere cavity, providing electric and magnetic field components. The prevailed electric component is vertically oriented, and the corresponding amplitude is close to $10^{-7} \mathrm{~V} / \mathrm{m}$. The magnetic field exhibits two horizontally potential components at N-S and E-W orientation with amplitudes of few tenths of picotesla. The detection of such weak electromagnetic fields in noisy environments is too difficult. Additionally, hardware imperfections can significantly reduce the performance of the system $[10,11]$. To improve the signal-to-noise ratio (SNR), it is necessary to use specialized sensors and electronic equipment [12]. Especially, in the ELF band, where Schumann resonances lie, very few works give details about measurement equipment used regarding electrical and magnetic antennas, the analog front-end, and the data acquisition module [13]. In the observation system of ULF/ELF emissions at Nakatsugawa, the signal observed by the N-S sensor (each coil consists of perm alloy of $1.2 \mathrm{~m}$ long with 100,000 turns of the copper wire) is fed 
to a preamplifier, then to a low-pass filter of 10 and $30 \mathrm{~Hz}$ and main-amplifier, stored on DL-708 data recorder and saved on a hard disk. Summarized values of the induction coils, the amplification, and filtering are given for the observation system of ULF/ELF emissions at Nakatsugawa [14]. Two research teams from Mexico have developed a Schumann resonance station with two inductive antennas. Details about the structure and development of the magnetic antenna are given. This station measures the first three harmonics [15]. In SR observatories in southwestern China, the frequency band of the instruments is about 3$29 \mathrm{~Hz}$ (in the range of $3 \mathrm{~dB}$ ), and the sampling frequency is $100 \mathrm{~Hz}$. There is a notch filter at $50 \mathrm{~Hz}$ to suppress industrial interference in the electronics part of [16]. Details and a block diagram, concerning associated electronics, of the TNB Antarctica Schumann measurement platform are presented by a research team from Italy [17]. In the Schumann station located at Calar Alto (Spain), modes are captured through the 2-m magnetic antenna with an acquisition time of $30 \mathrm{~min}$. Characterization of the sensor and detailed description of the amplification system are also presented [13]. The magnetometers, noise of the system, and the ELF measurement station that has been deployed in Sierra Nevada (Spain) are described and discussed by five research teams from Spain and Sweden [18]. The technical setup of the Hylaty geophysical station with a frequency range up to $300 \mathrm{~Hz}$, as well as the design of ELF equipment, including antennas and receivers, is discussed by three research teams from Poland [19]. In this paper, a versatile receiver for SR detection and monitoring is presented. The SR detection and monitoring system is portable, low-cost, battery-powered, autonomous for nearly 45 days' time, and able to measure up to six SR harmonics. The system, which consists of the induction coil antenna and the amplifying and filtering chain as well as the data acquisition and processing system, was designed and implemented entirely in the Electronics-Telecommunications and Applications Lab (ETA Lab) of the Physics Department of the University of Ioannina. The constructed magnetic field antenna is much smaller than the antennas described in the literature with cores $0.8-2 \mathrm{~m}$ long and a diameter of a few centimeters. The magnetic field antenna is implemented by two back-to-back identical induction coils [20] which are presented in detail and discussed in the following section. Moreover, the filtering and amplification chain exhibits an experimentally measured total passband gain equal to 410,000 or $112 \mathrm{~dB}$ (at $10 \mathrm{~Hz}$ ). The preliminary induction coil sensor antenna design has already been presented and described [21]. The design and implementation stages of the induction coil sensor were based on the fact that the induction core should avoid saturation due to external electromagnetic fields which were mostly originated from $50-\mathrm{Hz}$ power lines. Therefore, the SR measuring equipment was installed in relatively low "EM pollution" areas. In order to evaluate the portable SR system, measurements were acquired at various spots which were located at $1-\mathrm{km}$ far distances from man-made electromagnetic pollution in the area of Northwest Greece. From these measurements, it is obvious that up to six harmonics can be detected within 10-min acquisition time. Schumann resonance is a global phenomenon with numerous applications and many open questions [22]. As already mentioned, there is a very limited number of ELF measurement stations around the globe, based on synchronous electronic methodologies and techniques of signal reception, conditioning, and processing. The contribution of this new ELF Schumann resonance receiver includes (a) signal conditioning stages with an equivalent input noise as low as $2.88 \mathrm{nV} / \sqrt{\mathrm{Hz}}$ and a total passband gain from 86 to $112 \mathrm{~dB}$ at $10 \mathrm{~Hz}$, (b) monitoring and recording of six SR harmonics through a two back-to-back magnetic field antenna with total weight of $2.2 \mathrm{~kg}$ and $60 \mathrm{~cm}$ length, and (c) portability and over one and half month autonomy. Furthermore, discussion and detailed analysis of the efficient induction coil magnetic antenna and the low-noise amplifying-filtering chain through this work could motivate other researchers to create new and improved ELF measurement stations. The implemented induction coil antenna is presented and discussed in Section 2. The filtering and amplification chain we developed is extensively described in Section 3. Experimental results are present in Section 4, and finally, the article concludes with Section 5.

\section{Magnetic induction antenna}

Experimental measurements on Schumann resonance magnetic field components involve antenna implementations that are usually induction coils with efficient magnetic properties and enhanced detection sensitivity. Ferromagnetic core exhibits a relative magnetic permeability of the order of $10^{5}$, providing high induced voltage amplitude. Mumetal is a soft ferromagnetic material that exhibits a maximum magnetic permeability of the order of $2 \times 10^{5}$. Our antenna implementation is based on induction coil architecture with mumetal core material. That mumetal material (ASTM A753 Alloy 4) exhibits very small coercive force, very low core loss, and remanence. Due to the fact that our core is a rod that is open-ended, the resultant equivalent magnetic permeability is much smaller than the mumetal relative magnetic permeability and is given by Eqs. (1) and (2). Also, it strongly depends on the relative permeability of the core material as well as on the induction coil geometry through demagnetization factor $N$. Equations (1) and (2) 
give demagnetization factor $N$ and the resultant magnetic permeability $(\mu)$ of an induction coil.

$$
\begin{aligned}
& N=\frac{1}{m^{2}}[\ln (2 m)-1] \\
& \mu=\frac{\mu_{r}}{1+N\left(\mu_{r}-1\right)}
\end{aligned}
$$

where $m$ is the length to diameter core ratio ( $m=300$ / $25=12$ in this research) and $\mu_{r}$ is the relative magnetic permeability of the core material. In this work $N$ $=0.0151$.

In practice, choosing a quite large induction coil length is a benefit due to the resultant high magnetic permeability [23]. But taking into account that as the length of the induction coil increases, the ratio $m$ also increases eliminating the demagnetization factor value $N$, as observed from Eq. (1). That yields to the fact that the resultant magnetic permeability $(\mu)$ exhibits quite strong dependence on relative magnetic permeability $\left(\mu_{r}\right)$ of the core material due to Eq. (2). Moreover, the value of $\mu_{r}$ presents crucial instability resulting from temperature or applied field frequency variations. Therefore, the induction coil sensor performance may be crucially affected and degraded through variations of magnetic permeability $\mu$. As that material presents high relative magnetic permeability, Eq. 2 is modified to Eq. 3 and the induction coil magnetic permeability depends on the value of demagnetization factor $N$.

$$
\mu=\frac{1}{N} \quad(\mu=66 \text { in this research })
$$

Figure 1 depicts a block diagram of the single induction coil, and Table 1 summarizes the corresponding geometry values. The windings should be laid on the
Table 1 Single induction coil values

\begin{tabular}{ll}
\hline Geometrical parameter & Value \\
\hline ( $\left.I_{\text {core }}\right)$ : length of the ferromagnetic core (mumetal) & $300 \mathrm{~mm}$ \\
$\left(d_{\text {core }}\right)$ : diameter of the ferromagnetic core (mumetal) & $25 \mathrm{~mm}$ \\
$\left(d_{\text {wire }}\right)$ : diameter of the wire & $0.25 \mathrm{~mm}$ \\
$\left(I_{\text {winding }}\right)$ : length of the coil & $250 \mathrm{~mm}$ \\
$(t):$ thickness of the coil winding & $11 \mathrm{~mm}$ \\
$\left(d_{\text {total }}\right):$ diameter of the induction coil & $47 \mathrm{~mm}$ \\
$(n)$ : number of turns & 40,000 \\
$(\mu)$ & 66 \\
Weight & $2.2 \mathrm{~kg}$ \\
\hline
\end{tabular}

core within 70 to $90 \%$ of its total surface in order to take advantage of the maximum possible flux.

The self-inductance, resistance, and capacitance of the single induction coil were firstly computed through the calculated value of the demagnetization factor as well as the value of other geometry parameters and core material characteristics. Using Nagaoka's formula in the case of an air-coil inductor and a correction factor $\lambda$ which was proposed by Lukoschus [24] for core material inductors, the magnetic antenna self-inductance was calculated $[25,26]$. The induction coil resistance was then calculated through [27]. The magnetic antenna self-capacitance exhibited a strong dependence on the geometry of the coil as well as on the wire insulator electric permittivity and the shielding that may be used between the coil layers. Also, for the purpose of computing such a parameter, several extended computations [25] were needed. In practice, the induction coil self-capacitance was measured experimentally. Both calculated and measured values of the magnetic antenna self-inductance, resistance, and capacitance are summarized in Table 2.

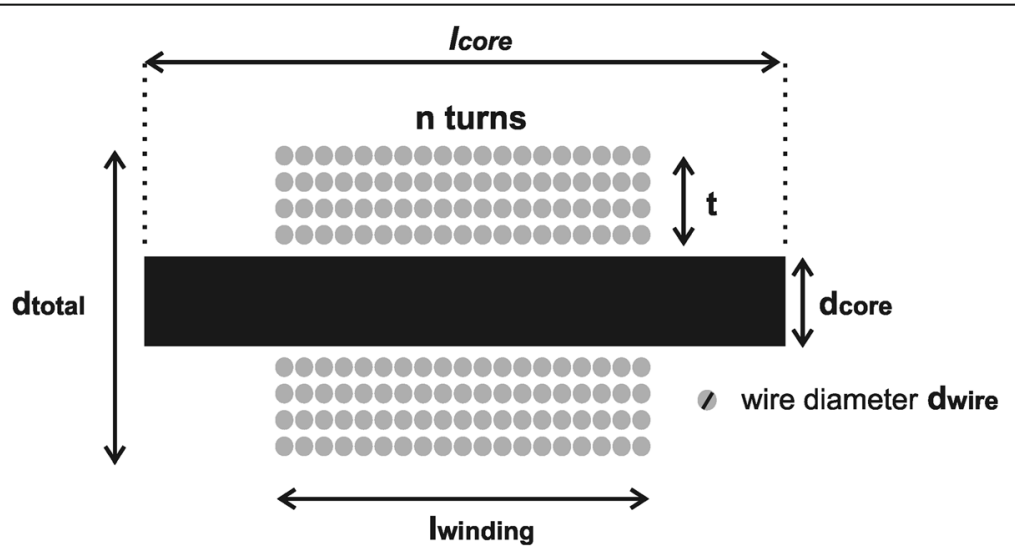

Fig. 1 Single induction coil geometry. Our induction coil architecture is depicted in such block diagram. The core mumetal material length ( $\left.I_{\text {core }}\right)$ and diameter $\left(d_{\text {core }}\right)$ as well as the length of the wire windings (I winding $)$ are also shown. We implement that induction coil antenna using copper wire (diameter- $d_{\text {wire }}$ ) and making $n$ turns of windings that cover $70-90 \%$ of the total core length. The total induction coil diameter is defined by the thickness of windings $(t)$ and the core diameter $\left(d_{\text {core }}\right)$, providing that the total number of turns should approximate to 40,000 
Table 2 Single induction coil $R, L$, and $C$ values

\begin{tabular}{lll}
\hline Parameters & Calculated values & Measured values \\
\hline L-inductance & 233 & $249 \mathrm{H}$ \\
R-resistance & 1548 & $1560 \Omega$ \\
$\mathrm{C}$ - capacitance & - & $440 \mathrm{pF}$ \\
\hline
\end{tabular}

The main self-resonance frequency $(1 / \sqrt{ } \mathrm{LC})$ of the single induction coil is $480 \mathrm{~Hz}$. However, as the bandwidth of the Schumann resonance frequency components lies in the ELF range, there is no risk of degradation on our induction coil's performance due to these self-resonances which are also filtered, efficiently, through our filtering and amplification chain stages. The implemented detection system includes two back-to-back coils (Fig. 2) resulting in a total coil length of $60 \mathrm{~cm}$. The housing of the induction coil antenna is provided by a Plexiglas container offering a versatile and portable setup for easy transportation and installation. The $60-\mathrm{cm}$ induction coil antenna (inside the Plexiglas container) is depicted in Fig. 2.

\section{Signal conditioning chain}

Analog front-end, regardless of its architecture, carries out filtering and amplification of received signals to the level required for the data acquisition module [28]. Specifically in this work, the filtering and amplification setup consists of six cascading circuits which provide amplification and filtering of the induced very low-amplitude voltage signal (few tens of $\mathrm{nV}$ ) coming out of the coil. Figure 3 shows a block diagram of the electronic chain. Our filtering and amplification chain consists of the following stages: preamplifier, low-pass filter and notch filter, double notch filter, double low-pass filter, high-pass filter, and variable gain amplifier. Since the maximum current of the signal condition setup does not exceed $120 \mathrm{~mA}$, one 47 -Ah battery with a 2.5-W solar battery charger extends autonomy for more than 45 days. Data acquisition and processing system has already been presented by Tatsis et.al [29] for the purpose of digitizing signals of the entire ELF band, with 16-bit resolution and an adjustable sampling rate.

For better noise performance and less signal degradation and distortion, the preamplifier was mounted directly on the coil inside the Plexiglas container as shown in Fig. 2.

\subsection{Preamplifier}

The first stage of the signal conditioning setup, which is the preamplifier, is shown in Fig. 4. The preamplifier stage is based on a non-inverted operational amplifier topology. The RC circuits at both $V_{\mathrm{CC}}$ and $V_{\mathrm{EE}}$ were used for decoupling purposes at both positive and negative supply voltages. Similar RC decoupling circuits were used in every stage of our filtering and amplification chain. The preamplifier's gain was equal to 35.5 , and the $-3-\mathrm{dB}$ cutoff frequency due to the RC filter in the output is $0.24 \mathrm{~Hz}$. In order to achieve additional low-noise performance, the OPA209 operational IC amplifier was chosen because it exhibits very good low-noise performance. For further investigation, we designed and implemented two more preamplifier stages that are based on inverted operational amplifier architecture and instrumentation amplifier topology, respectively. Taking into account our antenna coil architecture, we provided both simulated and measured results. These results indicated that both of those two alternative preamplifier stages had limited performance.

In order to evaluate the total rms equivalent input noise voltage of our preamplifier stage, we designed and implemented an equivalent input noise measurement platform. The test measurement platform consists of the following cascaded stages:

- The flat response amplifier with voltage gain of 392 .

- The second order band bass filter with passband voltage gain of 2.4, upper cutoff frequency of $52 \mathrm{~Hz}$, and lower cutoff frequency of $1.3 \mathrm{~Hz}$.

- The variable gain and flat response amplifier with voltage gain range from 1 to 21.

The maximum total gain of the three-stage amplification chain is $G_{\text {chain }}=19,757$. Feeding this chain with the

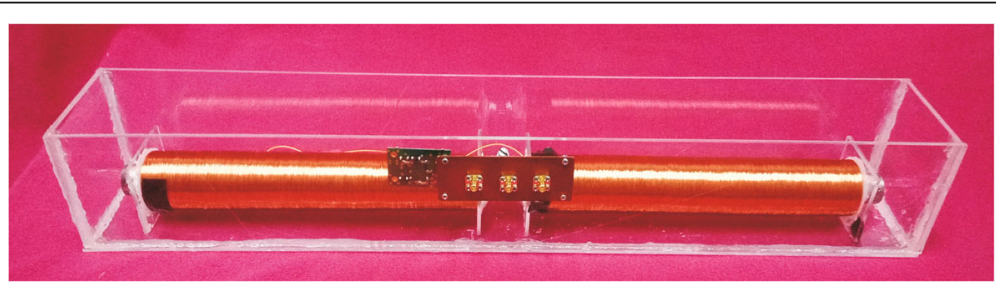

Fig. 2 Magnetic induction antenna. Our magnetic field antenna consists of two identical back-to-back induction coils with mumetal core material. In order to achieve core alignment and excellent connection between the two induction coils, we used a clamp of mumetal sheet. Our antenna output terminals are connected to the preamplifier inputs. A Plexiglas box is also used to protect the antenna and preamplifier stage mainly from moisture and humidity, providing also portability and stability. Through three SMA connectors, the preamplifier output as well as its positive and negative power supply is accessible 


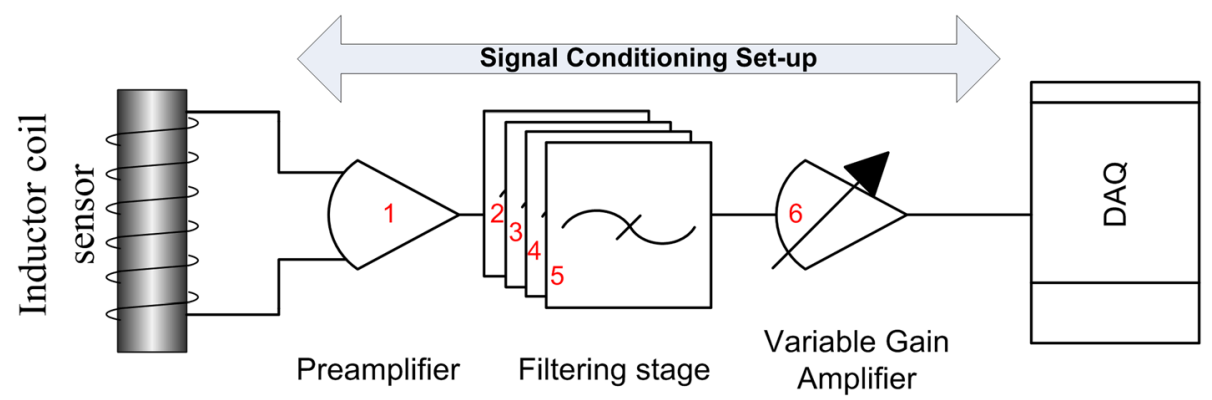

Fig. 3 Filtering and amplification chain. The architecture of our Schumann resonance detection and measuring system is shown in the schematic diagram. Two amplifying stages (preamplifier) and four active filtering stages are the signal conditioning setup that provide signal strength enhancement and bandwidth limitation in order to achieve Schumann resonance detection and measuring. The output of the last amplifying and filtering stage is connected to data acquisition system (DAQ). That system exploits digital signal techniques and methods, providing signal spectrum estimation and measuring

preamplifier's (PA) output, we obtain a total gain of $G_{\mathrm{PA}} \times G_{\text {chain }}=701,374$, where $G_{\mathrm{PA}}=35.5$. In order to measure the PA equivalent input noise, the preamplifier input is shorted to ground and the output is connected to the three-stage amplification chain. All these circuitries are installed inside a metallic box for electromagnetic protection and are battery-powered (Fig. 5a). Figure $5 \mathrm{~b}$ shows the noise obtained at the output of the above amplification chain where peak-to-peak value is $95 \mathrm{mV}$, considering that the amount of noise represents 99.904\% of the additive white Gaussian noise (AWGN) corresponding to the area between the probability density function (PDF) and the $\pm 3.3 \sigma$ limits.

Taking into account that the total passband gain is 701,374 , we deduce that the total rms equivalent input voltage noise is $20.5 \mathrm{nV}$ or $-154 \mathrm{dBV}$. This leads to an

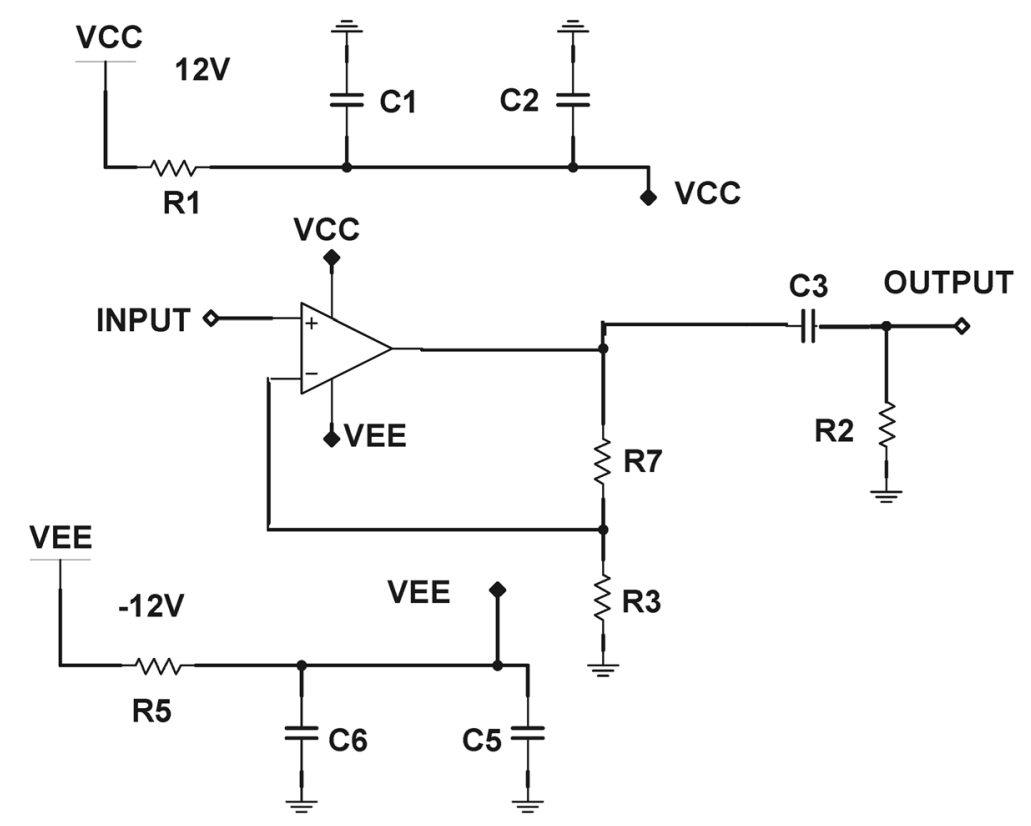

Fig. 4 Preamplifier. The first stage of our amplifying and filtering stage is based on non-inverted operational amplifier topology. The main goal is to provide low-noise amplification on the extra low-level-induced voltage at the induction coil terminals. Resistors R7 and R3 through feedback connection define the gain value as well as the output $R C$ filter stage $(R 2, C 3)$ eliminates flicker noise for better performance. We also used bypass capacitors at both positive and negative power supplies. The variable gain amplifier is the last stage of our amplifying and filtering chain. That stage is also based on non-inverted operational amplifier topology having a potentiometer in feedback instead of a fixed resistor value component. That potentiometer offers an efficient way to adjust total gain 

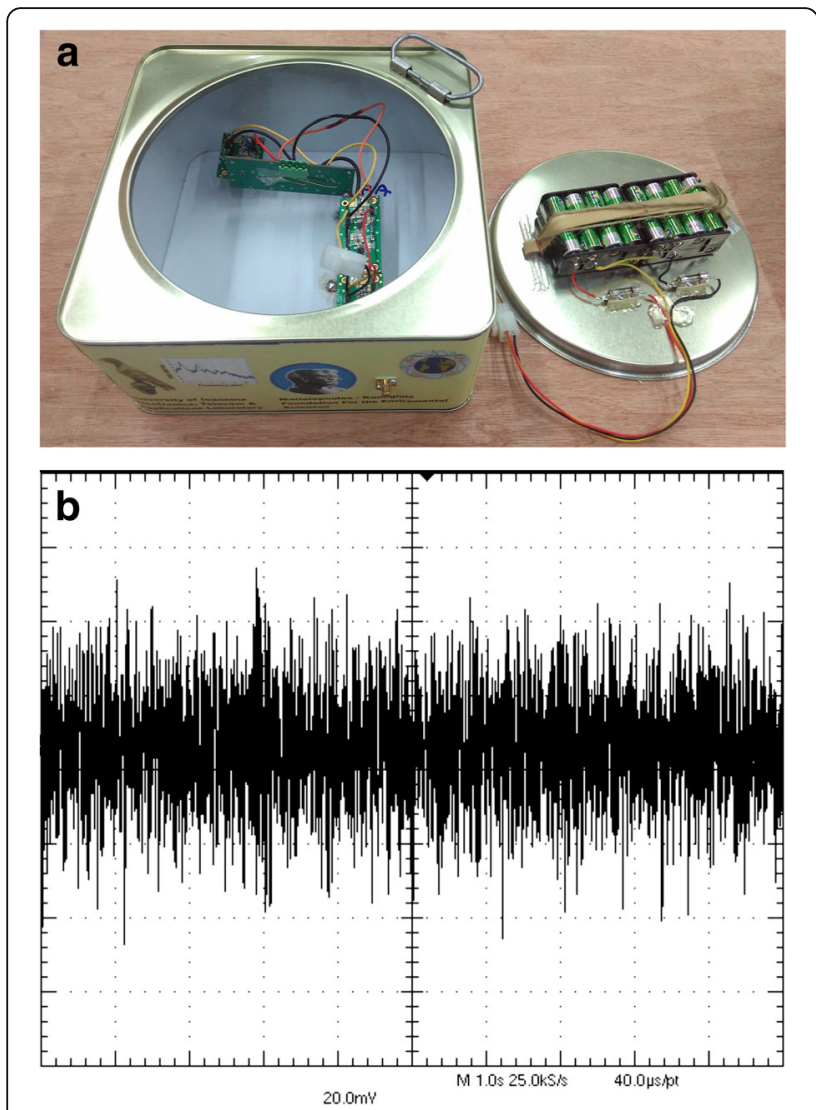

Fig. 5 a Metallic box for electromagnetic protection and batterypowered. b Experimental preamplifier voltage input noise, $20 \mathrm{mV} /$ division. For electromagnetic shielding, we used a metallic box that accommodates the under test preamplifier stage and the necessary amplifying and filtering stage for noise investigation. Also, we used batteries for power supply of the total circuitry. The SMA output provides noise measurements using digital oscilloscope. In order to achieve efficient estimation on equivalent input noise, the preamplifier inputs are shortened and the power supply is provided. Measuring the total output noise at several sequential snapshots, we made observations that confirm datasheet noise parameter value

equivalent input noise of $2.88 \mathrm{nV} / \sqrt{\mathrm{Hz}}$ for the bandwidth under consideration. This measured equivalent input noise of our preamplifier is in accordance with the equivalent input noise given by the manufacturer of OPA209 that is $3.3 \mathrm{nV} / \sqrt{\mathrm{Hz}}$ at $10 \mathrm{~Hz}$ and $2.25 \mathrm{nV} / \sqrt{\mathrm{Hz}}$ at $100 \mathrm{~Hz}$. This measured noise value is the worst-case noise appearing in the input, since our bandpass filter is not a brick wall but second-order Sallen-Key, and in addition, it includes any extra noise due to the $\mathrm{PCB}$, environment, etc. In addition to the equivalent input voltage noise, the PA exhibits also an input current noise that adds extra noise when flowing through the induction coil resistance. The total rms input current noise at bandwidth 1.3 to $52 \mathrm{~Hz}$ given by the manufacturer is $4.21 \mathrm{pA}$. A key element for efficient noise reduction of the entire SR measurement setup is to choose a preamplifier with equivalent input noise $\left(n_{\alpha}\right)$ as low as possible. This noise will be added orthogonally to the thermal noise of the coil $\left(n_{c}\right)$. In our case for $R_{\text {coil }}=3160 \Omega$, we obtain:

$$
n_{c}=\sqrt{4 k T R B}=50.92 n V
$$

Since the two noises $n_{a}$ and $n_{c}$ are orthogonal, we obtain total noise of the SR measurement setup:

$$
n_{t}=\sqrt{n_{\alpha}^{2}+n_{c}^{2}}=\sqrt{20.5^{2}+50.92^{2}} n V=54.9 n V
$$

which means that the preamplifier adds extra $0.65 \mathrm{~dB}$ noise on the resistive noise of the coil. When the PA is connected to the induction coil, the noise due to the input current noise will be only $13.9 \mathrm{nV}$. In the abovementioned noise measurement setup, the current noise contribution is zero since the input is shorted to ground.

\subsection{Filtering stage}

Our amplification and filtering chain has second to fourth stages that are based on Sallen-Key filter and Twin-T notch filter architectures. The Sallen-Key filter topology is a populated architecture mainly due to the fact that the filter performance exhibits very limited dependence on the operational amplifier performance. Also, the ratio of the largest passive component value to the smallest passive component value is very low. This is an important benefit in implementation. The Twin- $\mathrm{T}$ notch filter architecture offers versatile and efficient methods to design and implement band-stop filters using commercial operational amplifiers and standard values of passive components (resistors and capacitors). Moreover, using a resistor potentiometer at feedback line may provide adjustments that affect both the bandwidth and the resonance depth.

In particular, the second stage of our amplifying and filtering chain consists of a second-order Sallen-Key low-pass filter and a $50-\mathrm{Hz}$ Twin-T notch filter in cascading connection (Fig. 6). The passband gain of this stage is adjusted to 3.5 and the $-3-\mathrm{dB}$ cutoff frequency is $46 \mathrm{~Hz}$. The signal attenuation at $50 \mathrm{~Hz}$ is $10 \mathrm{~dB}$.

The third stage of the chain consists of two cascaded $50-\mathrm{Hz}$ Twin-T notch filters. The passband gain of this stage is 3.3 and the $-3-\mathrm{dB}$ cutoff frequency is $46 \mathrm{~Hz}$. The attenuation obtained at $50 \mathrm{~Hz}$ is $35 \mathrm{~dB}$.

The fourth stage of our amplifying and filtering chain is based on the fourth-order Sallen-Key low-pass filter topology. It has two second-order low-pass filters in cascading connection. The passband gain of this stage is 10 at the frequency range 1 to $45 \mathrm{~Hz}$, and the $3 \mathrm{~dB}$ cutoff frequency is $46 \mathrm{~Hz}$. 


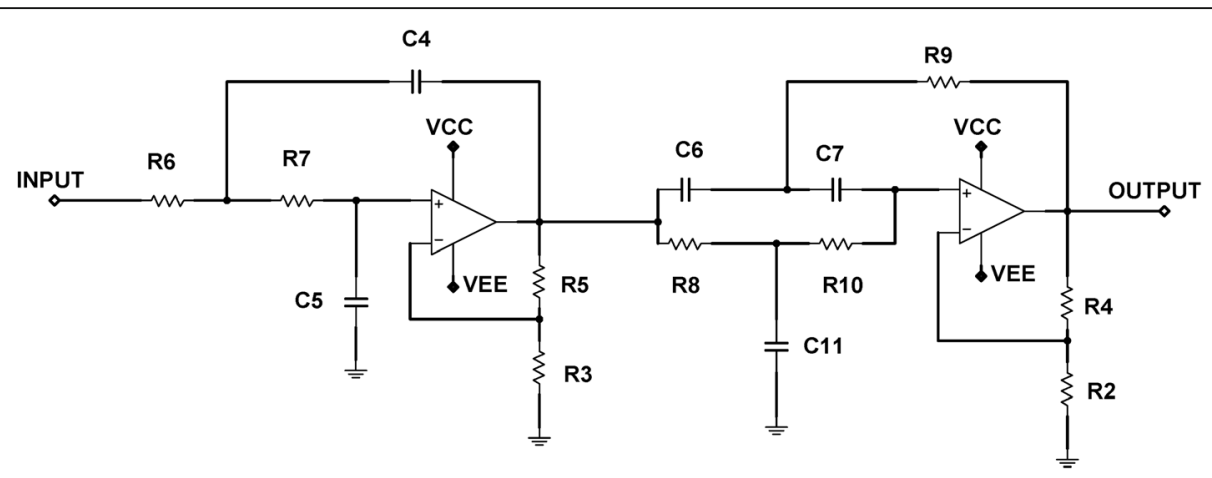

Fig. 6 Low-pass and notch filter. The schematic shows a second-order Sallen-Key low-pass filter and a Twin-T 50-Hz notch filter. These circuits are active filters providing amplification and signal bandwidth limitation mainly in the frequency range of $50 \mathrm{~Hz}$. Both the two architectures exhibit several advantages and remarkable performance in ELF range. The passive components needed to implement these circuits are commercially standard values and exhibit low-noise performance. Signal bandwidth and total gain requirements are taken into account

The fifth stage of our amplifying and filtering chain involves a Sallen-Key high-pass filter topology. The passband gain of this stage is 5 , while the $3-\mathrm{dB}$ cutoff is $1.8 \mathrm{~Hz}$.

\subsection{Variable gain amplifier}

The last stage (sixth) of our amplifying and filtering chain is a variable gain amplifier. It is based on non-inverting topology (similar to preamplifier topology). Both input and output have a passive single-pole RC high-pass filter with cutoff frequency $0.016 \mathrm{~Hz}$. These filters reduce the flicker noise, efficiently. The measured frequency response of this amplifier stage exhibits quite a flat response at the frequency range of interest ( 1 to $46 \mathrm{~Hz}$ ). The amplification of this stage ranges from 1 to 20 or adjustable through a potentiometer. Thus, the variable gain amplifier also offers a versatile way to adjust the total passband gain. That feature plays a key role on installing the portable Schumann resonance system at various environments due to adaptability.

\subsection{Total gain and response}

For more efficient electromagnetic shielding of all amplification and filtering stages, each stage is individually iron shielded as shown in Fig. 7. Moreover, all stages are mounted on a cup of a ferromagnetic box thus assuring additional shielding of the chain (Fig. 8), where the cup closes the metallic box. Table 3 summarizes the passband gain of each stages as well as the achieved total gain. These results are achieved through studies and investigation on all six stages in terms of performance and efficiency both using simulation and experimental measurements.

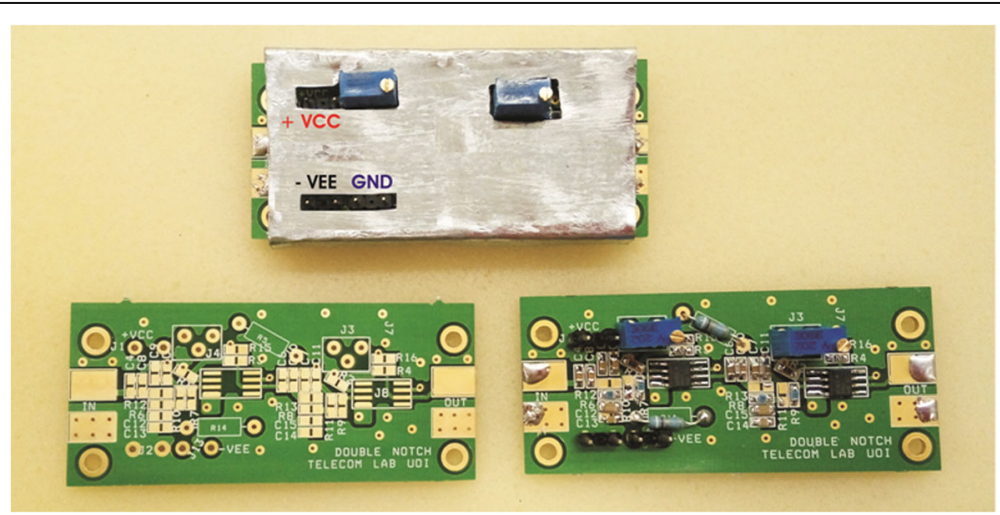

Fig. 7 Iron shielding of each stage, e.g., the double notch stage. The amplification and filtering chain stages are each arranged inside a metallic box for electromagnetic shielding. In order to achieve efficient noise performance, we designed and implemented such metallic boxes. These implementations provide electromagnetic shielding protection from any interference. Taking into account the extra low-level-induced voltage signal at antenna terminals, we should eliminate any source of interference that will provide signal degradation and distortion 


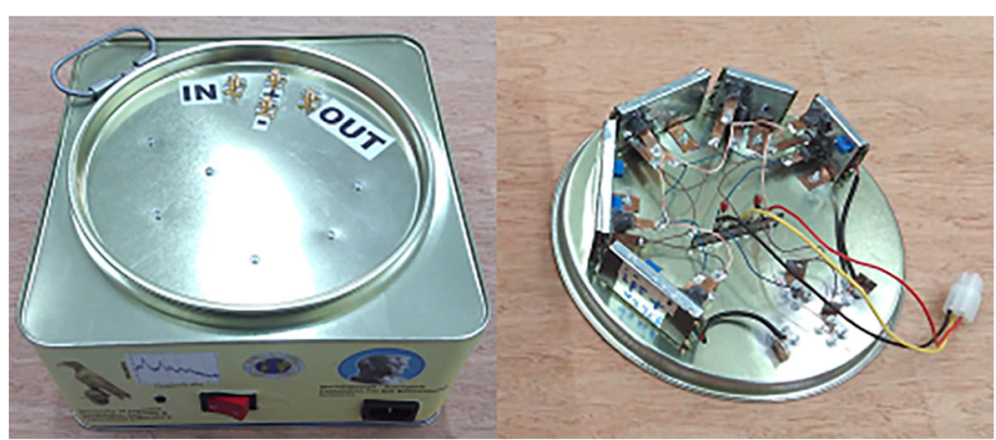

Fig. 8 Electromagnetic shielding metallic box. Apart from the preamplifier stage, the amplification and filtering stages (second to sixth) are all arranged inside a metallic box for electromagnetic shielding purposes. The total PCB circuits are mechanically adapted at the inner side of the box top. That arrangement allows us to adjust the total voltage gain through applying torque to the potentiometer screw at the variable gain amplifier. A connector at the outer side of the metallic box provides power supply at the amplification and filtering stages through an outer switch

The total simulated and measured frequency response of our amplifying and filtering chain is shown in Fig. 9, in case the passband gain of the variable gain amplifier has a prefixed value of 1 .

From these curves, it is obvious that there are limited declinations between the simulated and experimental results. These declinations are mostly observed at upper and lower cutoff frequencies ( 1 and $43 \mathrm{~Hz}$ ) as well as at $50 \mathrm{~Hz}$. The passive components (resistors, capacitors) we used exhibit limited parasitic resistances and reactances that may provide degradations on the total circuitry response. Also, the printed circuit board microstrip lines may have a possible impact on the total response results. In order to measure the signal chain response at the frequency range of $1-100 \mathrm{~Hz}$, we used our automated measurement setup that exhibits limitations on measuring and recording extra low amplitude signals. These limitations mainly provide the declinations between simulated and measured total response results at $50-\mathrm{Hz}$ frequency range.

Table 3 Signal conditioning stage passband gain values

\begin{tabular}{lll}
$\begin{array}{l}\text { Cascaded } \\
\text { stage number }\end{array}$ & Electronic circuit & $\begin{array}{l}\text { Passband gain value } \\
(\mathrm{dB}) \text { at } 10 \mathrm{~Hz}\end{array}$ \\
\hline 1 & Preamplifier & 35.5 \\
2 & $\begin{array}{l}\text { Low-pass and } \\
\text { notch filter }\end{array}$ & 3.5 \\
3 & Double notch & 3.3 \\
4 & $\begin{array}{l}\text { Double low-pass } \\
\text { filter }\end{array}$ & 10 \\
5 & High-pass filter & 5 \\
6 & $\begin{array}{l}\text { Voltage } \\
\text { gain amplifier }\end{array}$ \\
Total passband gain, 20501 to 410,020 (86 to 112) $\mathrm{dB}$ at $10 \mathrm{~Hz}$
\end{tabular}

For further investigation on the noise of the total system, we provided simulated results on equivalent input rms noise at each input of our amplifying and filtering chain stage (Table 4). At these simulation results, we assumed that the upper and lower limits of the noise bandwidth are 1 and $40 \mathrm{~Hz}$, respectively.

From these results, it is convenient that the total output noise of the implemented amplifying and filtering chain is mostly defined by the induction coil thermal noise and less by the preamplifier noise. In particular, experimental measurements on several outdoor environments indicate that voltage gain amplifier stage should have a passband gain of 4 for better performance on Schumann measuring and recording resonances. In that case, the equivalent rms input noise of the last amplifying and filtering chain stage approximates to $5135.3 \mathrm{nV}$. Besides, each system stage has its own impact on the total output noise. That impact is determined by each stage noise value and its sequential arrangement at our amplifying and filtering chain system. The last consideration is based on the fact that the stage voltage noise experiences lower gain and thus exhibits less effect on the total system noise as it arranges closer to the output of our chain.

Table 4 Noise analysis of signal chain blocks

\begin{tabular}{ll}
\hline Block description & Equivalent rms input noise (nV) \\
\hline Induction coil thermal noise & 44.7 \\
Preamplifier & 21.02 \\
Low-pass and notch filter & 1588.3 \\
Double notch filter & 1415 \\
Double low-pass filter & 3977.5 \\
High pass filter & 1287 \\
Voltage gain amplifier & 555.75 to $1482.1 \times 10^{3}$ \\
\hline
\end{tabular}




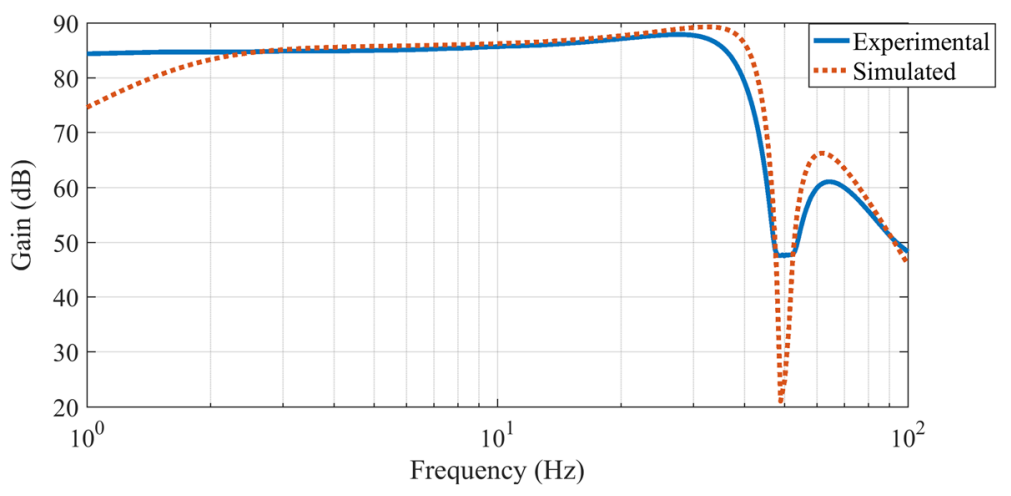

Fig. 9 Simulated and experimental frequency response of filtering and amplifying system. In order to make efficient investigation on the total amplification and filtering chain, we provide simulated and measured results. These are frequency response results that help us to make useful output signal level and bandwidth considerations. Both simulated and measured results provide a flat frequency response at the frequency range of interest. Also, these results indicate that the $50-\mathrm{Hz}$ power line signal component exhibits extra low level that eliminates any output signal degradation and distortion mainly due to induction coil saturation effects

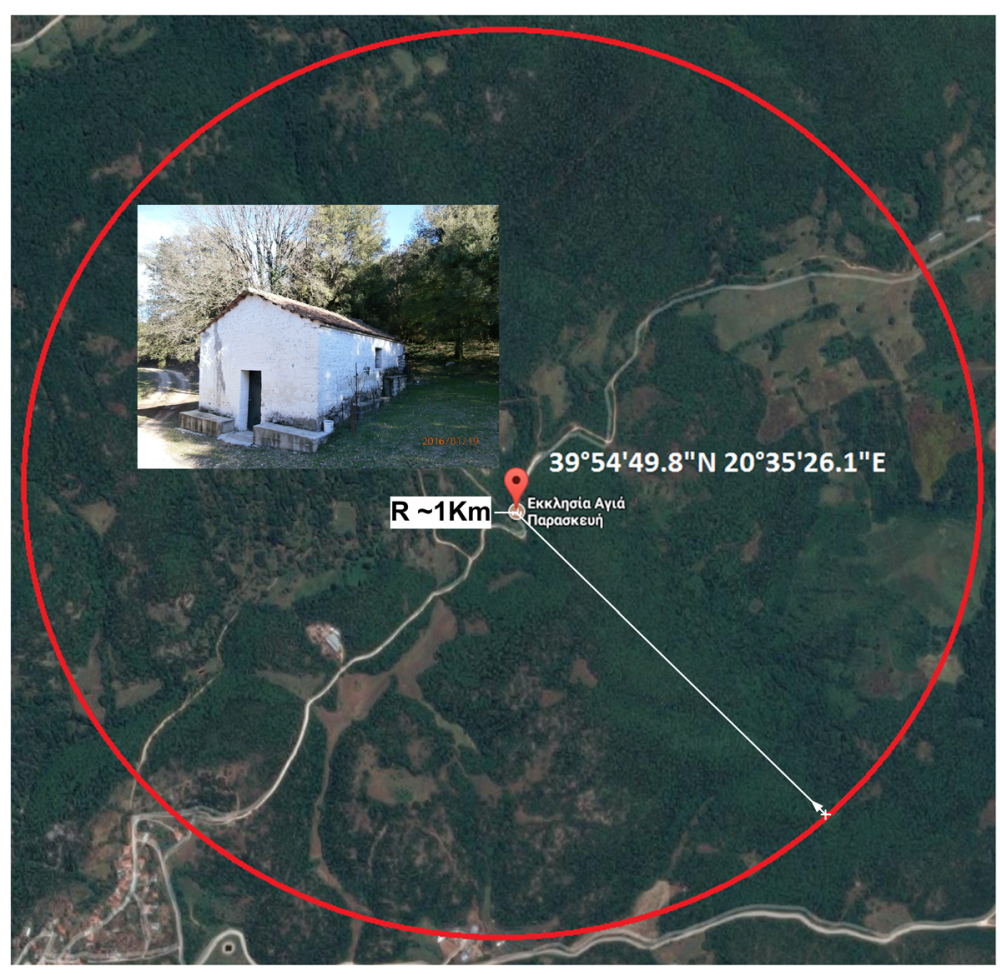

Fig. 10 Long-term measurement spot. At nearly $1 \mathrm{~km}$ far away from the Doliana village (loannina, Greece), there is a chapel. That building provides an efficient place in order to accommodate our Schumann resonance detection and measuring system at a non-EM pollution environment. We need $40 \mathrm{~min}$ in order to visit that place by car. The power line network is not accessible from that building. Any other houses and buildings are far away from that spot. At that chapel neighborhood, any human activities are nearly rare. These features offer an extra low-noise environment with low level electromagnetic pollution, making that place efficient and very attractive for Schumann resonances measurements 
Table 5 Noise analysis of signal chain blocks

\begin{tabular}{ll}
\hline Block description & Noise figure $(\mathrm{dB})$ at $10 \mathrm{~Hz}$ \\
\hline Preamplifier & 11.76 \\
Low-pass and notch filter & 49.02 \\
Double notch filter & 45.89 \\
Double low-pass filter & 57.18 \\
High-pass filter & 36.71 \\
Voltage gain amplifier & 27.77 to 104.62
\end{tabular}

We also provide simulated results on noise figure (NF) in decibels at $10 \mathrm{~Hz}$ for each stage of our amplifying and filtering chain (Table 5).

\section{Measurements on the field-results}

In order to confirm and improve the system's operation, we made short-term measurements (few hours) mainly in "almost" white zones in the region of Epirus (NW Greece). Those spots as well as geographical coordinates and distance from the EM pollution are shown on Table 6. Except the last spot (chapel at Doliana), all the other places are in the countryside not suitable for long-term housing of our SR detection and monitoring system. Finally, the measurement equipment have been installed at the fifth spot, a chapel located at a small gorge, $570 \mathrm{~m}$ above the sea level and around $1 \mathrm{~km}$ away from man-made electromagnetic pollution (Fig. 10). The SR system starts measuring on 19 January 2016 with concurrent recordings, with few time gaps due to electronic equipment maintenance and local interference. Since June 2016, ETA Lab's SR measurement station has been added to the list of worldwide Schumann resonance stations [30].

To verify that the primary aim of this work was achieved, which was to design and implement a standalone, portable, and low-cost system able to

Table 6 Short- and long-term measurement spots

\begin{tabular}{|c|c|c|c|}
\hline Spots & Lat/long & $\begin{array}{l}\text { Distance from EM } \\
\text { pollution }(\mathrm{km})\end{array}$ & $\begin{array}{l}\text { Altitude } \\
\text { (m) }\end{array}$ \\
\hline 1. Konitsa & $\begin{array}{l}40^{\circ} 04^{\prime} 51.9^{\prime \prime} \\
\text { N } 20^{\circ} 45^{\prime} 39.4^{\prime \prime} \text { E }\end{array}$ & 1.0 & 808 \\
\hline 2. Neochori & $\begin{array}{l}39^{\circ} 02^{\prime} 29.4^{\prime \prime} \\
N 20^{\circ} 59^{\prime} 00.8^{\prime \prime} E\end{array}$ & 2.8 & 1 \\
\hline 3. Politsas bridge & $\begin{array}{l}39^{\circ} 30^{\prime} 38.3^{\prime \prime} \\
N 21^{\circ} 00^{\prime} 31.3^{\prime \prime} E\end{array}$ & 2.5 & 302 \\
\hline 4. Filiates & $\begin{array}{l}39^{\circ} 37^{\prime} 58.3^{\prime \prime} \\
N 20^{\circ} 15^{\prime} 07.9^{\prime \prime} E\end{array}$ & 1.6 & 144 \\
\hline 5. Chapel at Doliana & $\begin{array}{l}39^{\circ} 54^{\prime} 49.8^{\prime \prime} \\
\text { N } 20^{\circ} 35^{\prime} 26.1^{\prime \prime} \mathrm{E}\end{array}$ & 1.0 & 570 \\
\hline
\end{tabular}
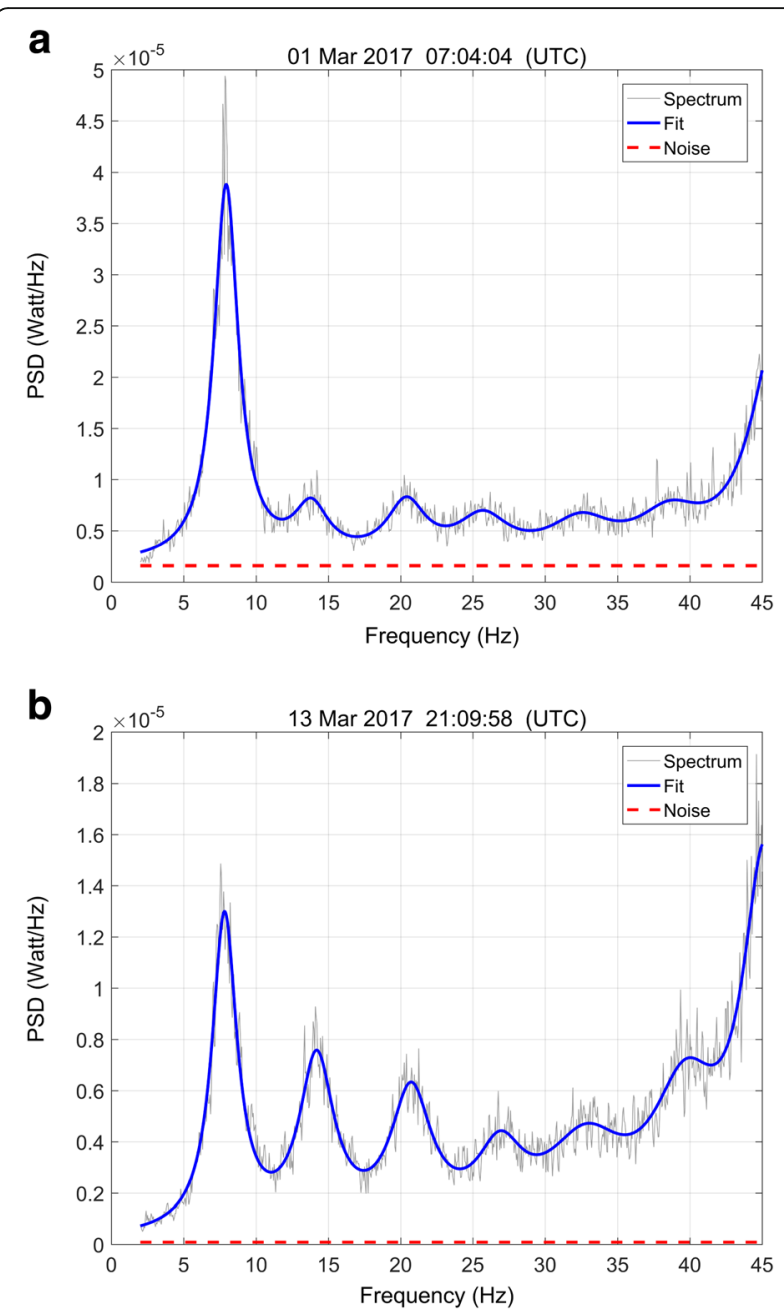

Fig. 11 Power spectrum of recorded signal 10-min snapshot. Our induction coil system having two identical back-to-back magnetic antennas aligned at $\mathrm{N}-\mathrm{S}$ orientation offer a big amount of measurements at 10-min duration acquisition time intervals. Two of such snapshots are depicted in Fig. 11. Not only the measured results but also the noise and fitting curves based on nonlinear least square regression, using Lorentzian-like function, are shown. From these curves, we observed six Schumann resonance modes and their parameters that relate to resonant frequencies, $Q$ factors, and peak powers. At the top of $\mathbf{a}$ and $\mathbf{b}$, there is the detailed date and time of the measurement snapshot

Table 7 SR data

\begin{tabular}{llllllll}
\hline Mode & \multicolumn{3}{l}{ 01 Mar 2017 07:04:04 (UTC) } & & \multicolumn{4}{c}{ 13 Mar 2017 21:09:58 (UTC) } \\
\cline { 2 - 3 } & $f(\mathrm{~Hz})$ & $P_{\mathrm{m}}(\mathrm{mW} / \mathrm{Hz})$ & $\mathrm{Q}$ & & $f(\mathrm{~Hz})$ & $P_{\mathrm{m}}(\mathrm{mW} / \mathrm{Hz})$ & $\mathrm{Q}$ \\
\hline 1 & 7.93 & 0.0367 & 3.88 & & 7.81 & 0.0123 & 3.72 \\
2 & 13.81 & 0.0049 & 4.73 & & 14.18 & 0.0066 & 4.82 \\
3 & 20.39 & 0.0053 & 6.18 & 20.72 & 0.0052 & 5.96 \\
4 & 25.69 & 0.0039 & 5.88 & 26.87 & 0.0027 & 6.81 \\
5 & 32.44 & 0.0034 & 6.08 & 32.78 & 0.0032 & 4.92 \\
6 & 38.60 & 0.0038 & 7.08 & 39.68 & 0.0049 & 7.12 \\
\hline
\end{tabular}


Table 8 SR mean values and standard deviations, May 2017

\begin{tabular}{lll}
\hline Mode & $\bar{f}(\mathrm{~Hz})$ & $\sigma_{f}(\mathrm{~Hz})$ \\
\hline 1 & 7.70 & 0.46 \\
2 & 14.43 & 0.59 \\
3 & 20.74 & 0.46 \\
4 & 26.57 & 0.84 \\
5 & 33.23 & 0.77 \\
6 & 40.01 & 1.27 \\
\hline
\end{tabular}

measure as much SR harmonics as possible, we present power spectrums with $60 \mathrm{~cm}$ back-to-back magnetic antenna and N-S orientation with 10-min acquisition time (Fig. 11). Each figure additionally contains noise and fitting curves based on nonlinear least square regression, using Lorentzian-like function as described at [29]. For the first six modes, resonant frequencies, $Q$ factors, and peak powers are summarized in Table 7. Although detailed analysis and validation of the record data is beyond the scope of this work, additional results are presented to document the system's operability. Specifically, frequency mean value and standard deviation for the first six modes and for May 2017 are shown on Table 8. Frequency variation versus time, for the first week of May 2017, for mode 1 is shown in Fig. 12. Daily frequency variation, with maximum values around $(8.00 \pm 0.05) \mathrm{Hz}$ for the first 4 days and $(7.90 \pm 0.05) \mathrm{Hz}$ for the last 3 days, is noticed. Respectively, minimum frequency values lie around
$(7.75 \pm 0.05) \mathrm{Hz}$ and $(7.55 \pm 0.05) \mathrm{Hz}$. For all 7 days, maximum frequency values occur between 2:00 am and 6:00 am while minimum frequency values occur between 11:00 am and 3:00 pm, which yields similar frequency patterns for mode 1 . These day-to-night and day-to-day variations are interpreted mainly by the inhomogeneities and the anisotropy in the Earth-ionosphere waveguide.

\section{Conclusions}

A new portable ELF receiver for Schumann resonance detection and monitoring is presented in detail in this paper. The system is comprised of a self-designed induction coil sensor and six filtering and amplification stages. In the presented system, two magnetic field antennas, with a weight of $2.2 \mathrm{~kg}$ and size of $300 \times 25 \mathrm{~mm}$ each were developed. The main self-resonance frequency of the single induction coil is $480 \mathrm{~Hz}$. The low-noise implemented signal conditioning stages have an equivalent input noise as low as $2.88 \mathrm{nV} / \sqrt{ } \mathrm{Hz}$ and a total passband gain from 86 to $112 \mathrm{~dB}$ at $10 \mathrm{~Hz}$. The experimental results have also demonstrated the system's feasibility in measuring clearly up to the sixth SR harmonic. Since January 2016, the measurement system has been installed near North-West borders of Greece measuring and storing SR initially with one magnetic coil and since June 2016 with two magnetic coils back to back. The system's remote monitoring, validation of 18 months of data, and installation of E-W magnetic coil antenna are included in a short-term future work.

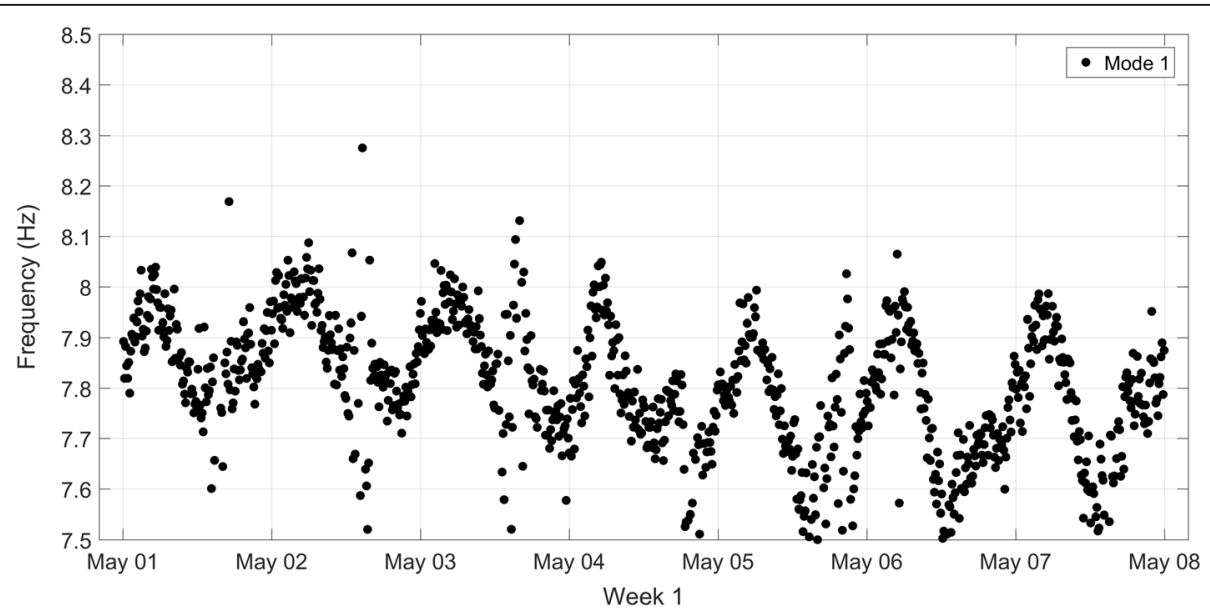

Fig. 12 Frequency variation over a week for mode 1. The fundamental Schumann resonance mode (at 7.8 frequency range) exhibits not only resonance frequency variation but also $Q$ factors and peak powers during a day, giving interesting and important observations that motivate further studying and investigation. In Fig. 12, the resonance frequency variation results of the fundamental Schumann resonance mode are shown. The weekly duration of such results indicate that the first mode of the Schumann resonance spectrum exhibits a slow daily variation that ranges from 7.5 to $8.1 \mathrm{~Hz}$ caused mainly by the inhomogeneities and the anisotropy in the Earth-ionosphere waveguide 


\section{Acknowledgements}

The authors would like to thank the retired principal of Doliana's senior high school Mr. G. Skordos and the Ecclesiastical Committee for indicating places with low man-made electromagnetic radiation. Also, they offer a special word of thanks to Mr. A. Tsolis for his granting permission to install our measurement setup at his low-level electromagnetic field spot located in Neochori, Arta, Greece. We would also like to thank Empirikion Foundation for their financial support.

\section{Funding}

This research was supported by the Mariolopoulos-Kanaginis Foundation for the Environmental Sciences, grant no.119/20.04.2012.

\section{Availability of data and materials}

All the presented data are fully available without restriction.

\section{Authors' contributions}

All authors have contributed in a variety of ways to this work. All authors read and approved the submitted manuscript.

\section{Competing interests}

The authors declare that they have no competing interests.

\section{Publisher's Note}

Springer Nature remains neutral with regard to jurisdictional claims in published maps and institutional affiliations.

\section{Author details}

'Electronics-Telecommunications and Applications Lab Physics Department, University of loannina, 45110 loannina, Greece. ${ }^{2}$ Mariolopoulos-Kanaginis Foundation for the Environmental Sciences, 30-32 Patriarchou loakim Str, 106 75 Athens, Greece.

\section{Received: 3 January 2018 Accepted: 30 May 2018}

\section{Published online: 18 June 2018}

\section{References}

1. A Salinas, S Toledo-Redondo, EA Navarro, J Fornieles-Callejón, JA Portí, Solar storm effects during Saint Patrick's Days in 2013 and 2015 on the Schumann resonances measured by the ELF station at Sierra Nevada (Spain). J. Geophys. Res. Space Physics 121(12), 12,234-12,246 (2016). https://doi.org/10.1002/2016ja023253

2. A Shvets, M Hayakawa, Global lightning activity on the basis of inversions of natural ELF electromagnetic data observed at multiple stations around the world. Surv. Geophys. 32(6), 705-732 (2011) https://doi.org/10.1007/s10712011-9135-1

3. H Zhou, X Qiao, Studies of the variations of the first Schumann resonance frequency during the solar flare on 7 March 2012. Journal of Geophysical Research: Atmospheres 120(10), 4600-4612 (2015)

4. E Greenberg, C Price, Y Yair, M Ganot, J Bór, G Sátori, ELF transients associated with sprites and elves in eastern Mediterranean winter thunderstorms. J. Atmos. Sol. Terr. Phys. 69(13), 1569-1586 (2007). https:// doi.org/10.1016/j.jastp.2007.06.002

5. J Kozakiewicz, A Kulak, J Mlynarczyk, Analytical modeling of Schumann resonance and ELF propagation parameters on Mars with a multi-layered ground. Planetary and Space Science 117, 127-135 (2015). https://doi.org/ 10.1016/j.pss.2015.05.019.

6. Sekiguchi, Hayakawa, Nickolaenko, and Hobara. Evidence on a link between the intensity of Schumann resonance and global surface temperature. Ann. Geophys. 24, no. 7 (2006), 1809-1817. doi:https://doi.org/10.5194/angeo-241809-2006.

7. F Simões, M Rycroft, N Renno, Y Yair, KL Aplin, Y Takahashi, Schumann resonances as a means of investigating the electromagnetic environment in the solar system. Space Sciences Series of ISSI, 455-471 (2008). https://doi. org/10.1007/978-0-387-87664-1_28

8. ER Williams, The Schumann resonance: a global tropical thermometer Science 256(5060), 1184-1187 (1992). https://doi.org/10.1126/science.256. 5060.1184

9. H Zhou, Z Zhou, X Qiao, H Yu, Anomalous phenomena in Schumann resonance band observed in China before the 2011 magnitude 9.0 Tohoku-
Oki earthquake in Japan. Journal of Geophysical Research: Atmospheres 118(23), 13,338-13,345 (2013)

10. A Balatsoukas-Stimming et al., Baseband and RF hardware impairments in full-duplex wireless systems: experimental characterisation and suppression. EURASIP J. Wirel. Commun. Netw. 2015(1) (2015). https://doi.org/10.1186/ s13638-015-0350-1

11. M Ariaudo et al., Green radio despite "Dirty RF" front-end. EURASIP J. Wirel. Commun. Netw. 2012(1) (2012)

12. P Dolea, V Dascal, T Palade, O Cristea, Low-cost prototype equipment for vlf radio monitoring. ACTA TECHNICA NAPOCENSIS (Electronics and Telecommunications) 53(1), 47-51 (2012) Retrieved from http://users.utcluj. ro/ atn/

13. JA Parra, MF Ros, NN Castellano, RM Salvador, Techniques for Schumann resonance measurements: a comparison of four amplifiers with a noise floor estimate. IEEE Trans. Instrum. Meas. 64(10), 2759-2768 (2015)

14. K Ohta, K Umeda, N Watanabe, M Hayakawa, ULF/ELF emissions observed in Japan, possibly associated with the Chi-Chi earthquake in Taiwan. Natural Hazards and Earth System Science 1(1/2), 37-42 (2001)

15. FP Sierra, HS Vazquez, ME Andrade, B Mendoza, D Rodriguez-Osorio, Development of a Schumann-resonance station in Mexico: preliminary measurements. IEEE Antennas and Propagation Magazine 56(3), 112-119 (2014)

16. X Ouyang, X Zhang, AP Nickolaenko, M Hayakawa, X Shen, Y Miao, Schumann resonance observation in China and anomalous disturbance possibly associated with Tohoku M9.0 earthquake. Earthq. Sci. 26(2), $137-$ 145 (2013). https://doi.org/10.1007/s11589-013-0009-0

17. C Rossi, P Palangio, F Rispoli, Investigations on diurnal and seasonal variations of Schumann resonance intensities in the auroral region. Ann. Geophys. 50(3), 301-311 (2007)

18. J Fornieles-Callejón, A Salinas, S Toledo-Redondo, J Portí, A Méndez, EA Navarro, JA Morente-Molinera, C Soto-Aranaz, JS Ortega-Cayuela, Extremely low frequency band station for natural electromagnetic noise measurement Radio Sci. 50(3), 191-201 (2015)

19. A Kulak, J Kubisz, S Klucjasz, A Michalec, J Mlynarczyk, Z Nieckarz, et al., Extremely low frequency electromagnetic field measurements at the Hylaty station and methodology of signal analysis. Radio Sci. 49(6), 361-370 (2014) https://doi.org/10.1002/2014rs00540

20. Shvets, A. (Private communication, March 2016)

21. C Votis, G Tatsis, V Christofilakis, P Kostarakis, V Tritakis, C Repapis, Design and implementation of Schumann resonances sensor platform. Journal of Engineering Science and Technology Review 9(6), 112-115 (2016) Retrieved from http://www.jestr.org/downloads/Volume9|ssue6/fulltext16962016.pdf

22. C Price, ELF electromagnetic waves from lightning: the Schumann resonances. Atmosphere 7(9), 116 (2016) https://doi.org/10.3390/ atmos7090116

23. Sgobba, S. (2009). Physics and measurements of magnetic materials. CERN2010-004, 39-63. Retrieved from arXiv:1103.1069v1

24. D Lukoschus, Optimization theory for induction-coil magnetometers at higher frequencies. IEEE Trans. Geosci. Electron. 17(3), 56-63 (1979)

25. C Christophe, L Paul, Induction magnetometers principle, modeling and ways of improvement (INTECH Open Access Publisher, 2012)

26. S Tumanski, Induction coil sensors - a review. Meas. Sci. Technol. 18(3), R31-R46 (2007)

27. S Macintyre, in Webster, ed. by G Chief John. The measurement, instrumentation and sensors handbook (1999)

28. YS Poberezhskiy, GY Poberezhskiy, Flexible analog front ends of reconfigurable radios based on sampling and reconstruction with internal filtering. EURASIP J. Wirel. Commun. Netw. 2005(3), 381832 (2005)

29. G Tatsis, C Votis, V Christofilakis, P Kostarakis, V Tritakis, C Repapis, A prototype data acquisition and processing system for Schumann resonance measurements. J. Atmos. Sol. Terr. Phys. 135, 152-160 (2015)

30. Williams, E. R. (Private communication, June 2016). 